\title{
A Case of Sudden Diffuse Thyroid Swelling after Fine Needle Aspiration Cytology where Changes over Time Were Confirmed by Ultrasonography
}

\author{
Naoki Takemoto ${ }^{1,2}$ Taijiro Ozawa ${ }^{2}$ Hitomi Tamai ${ }^{2}$ \\ ${ }^{1}$ Department of Otolaryngology, Head and Neck Surgery, Nagoya \\ City University, Nagoya, Japan \\ 2 Department of Otolaryngology, Toyohashi Municipal Hospital, \\ Toyohashi, Japan \\ Int J Pract Otolaryngol 2021;4:e1-e5.
}

\author{
Tatsuhiro Asaoka ${ }^{2}$ Shinichi Iwasaki ${ }^{1}$
}

\begin{abstract}
Address for correspondence Naoki Takemoto, MD, Medical Office, Department of Otolaryngology, Head and Neck Surgery, Kawasumi 1, Mizuhocho, Mizuho Ward, Nagoya, 467-8601 Aichi, Japan (e-mail: naoki.takemoto.g@gmail.com).
\end{abstract}

\begin{abstract}
Keywords

- diffuse thyroid swelling

- fine needle aspiration

- ultrasonography

- complication

We encountered a case of diffuse thyroid swelling after fine needle aspiration (dTSaFNA) cytology, and monitored the swelling by thyroid ultrasonography. The scan findings are reported. A 57-year-old female visited our hospital complaining of swelling in her anterior neck. She had a history of metal allergy and was not taking any medications. Because a solid cystic tumor was detected in the left thyroid lobe, we decided to perform fine-needle aspiration cytology (FNAC). When the second aspiration was performed with a $22 \mathrm{G}$ needle inserted into the nodule in the left lobe, the patient immediately complained of pain in her neck. Within 2 minutes, her anterior neck swelled up and ultrasonography showed vascular dilatation in the left thyroid lobe. Neck cooling with steroid injection was undertaken. However, over the next 5 minutes, the vascular dilatation also spread to the right lobe. About 15 minutes later, the vessels gradually became unclear, and the neck pain and swelling persisted, and the patient was hospitalized for observation. By the following day, all the symptoms and signs had resolved and the patient was discharged. FNA is associated with the risk of neck swelling caused by hemorrhage or DTSaFNA. To make a prompt diagnosis and provide appropriate treatment for this complication, ultrasonography could be useful.
\end{abstract}

\section{Introduction}

Fine needle aspiration cytology (FNAC) for thyroid nodules is particularly important for routine examination in otolaryngology dealing with the thyroid. Neck swelling, a rare complication of FNAC, is caused by hematoma formation because of bleeding from the puncture site; diffuse thyroid swelling is caused by intraglandular vasodilation induced by puncture stimulation., ${ }^{1,2}$ While an appropriate name for diffuse thyroid swelling has yet to be determined, Ohtsuka et al referred to it as diffuse thyroid swelling after fine needle aspiration (dTSaFNA). ${ }^{3}$ Moreover, we used the term dTSaFNA in this study for convenience. We observed dTSaFNA changes over time using ultrasonography and obtained their characteristics.

\section{Case Presentation}

\section{Patient}

A 57-year-old female.

\section{Chief Complaint}

Thyroid nodule. received

May 11,2020

accepted

August 13, 2020
DOI https://doi.org/

10.1055/s-0040-1722335. ISSN 2569-1783.

\footnotetext{
(C) 2021. The Author(s).

This is an open access article published by Thieme under the terms of the Creative Commons Attribution-NonDerivative-NonCommercial-License, permitting copying and reproduction so long as the original work is given appropriate credit. Contents may not be used for commercial purposes, or adapted, remixed, transformed or built upon. (https://creativecommons.org/ licenses/by-nc-nd/4.0/) Georg Thieme Verlag KG, Rüdigerstraße 14, 70469 Stuttgart, Germany
} 


\section{General History}

Allergic to various metals with no history of drug treatment for allergies.

\section{History of Present Illness}

As a nodule was identified in the left thyroid lobe using ultrasonography during an emergency outpatient visit for palpitation, the patient visited the Department of Otolaryngology as an outpatient the following day. There were no symptoms during consultation at our department.

\section{Symptoms at Initial Examination}

A movable mass with slight induration was observed at the anterior neck; there was no tenderness.

\section{Laboratory Findings}

Laryngeal endoscopy demonstrated no vocal cord paralysis, blood test showed thyroid-stimulating hormone of $0.983 \mu \mathrm{IU} / \mathrm{mL}$, fT3 of $3.24 \mathrm{pg} / \mathrm{mL}$, and fT4 of $1.17 \mathrm{ng} / \mathrm{mL}$, thus indicating normal thyroid function. Ultrasonography revealed a well-defined mass of $22.3 \times 16.3 \times 24.7 \mathrm{~mm}$ with microcalcification inside (-Fig. 1). FNAC was performed on the nonfunctional thyroid nodule.

\section{Fine Needle Aspiration Cytology}

In terms of procedure, a $22 \mathrm{G}$ needle, a $10-\mathrm{mL}$ syringe, and an extension tube were connected. Moreover, negative pressure suction was performed for approximately $5 \mathrm{~mL}$ after puncture. The first FNA was completed without any issue; however, there was no sample attached to the glass slide, and thus we decided to perform puncture again. Immediately after the second FNA ( - Fig. 2a), the patient complained of pain at the puncture site, and FNA was discontinued. The pain remained even after removal and general swelling of the anterior neck was observed. Moreover, 2 minutes after the second FNA (-Fig. 2b), ultrasonography revealed intrathyroid vasodilation in both thyroid lobes. Vasodilation was more prominent around the left lobe nodule. Assuming dTSaFNA, anterior neck cooling and $200 \mathrm{mg}$ of intravenous drip of hydrocortisone sodium succinate (Solu-Cortef) were performed. Furthermore, 5 minutes after the second FNA (-Fig. 2c), ultrasonography was performed again. This revealed slightly vague vasodilation of the punctured left lobe, while vasodilation of the contralateral right lobe became most prominent. Moreover, 15 minutes after the second FNA( - Fig. 2d), vasodilation was vague in both lobes, but anterior neck swelling continued. Laryngeal endoscopy demonstrated no laryngeal edema and airway stenosis because of compression. Because the anterior neck swelling ( - Fig. 3) and pain continued, the patient was hospitalized for observation, and the swollen thyroid was marked with a marker pen. Then, the day after the first visit to our department, swelling and pain improved, and the patient was discharged. Subsequently, 5 days after the first visit to our department, FNAC results demonstrated unsuitable samples. A wheal matching the marking appeared during consultation. As the patient did not wish to undergo further diagnosis and treatment through reexamination and surgery, we decided to perform follow-up with ultrasonographic observation. At the current follow-up time point of approximately 1.5 years, there have not been any changes in lesion size or characteristics.

\section{Discussion}

Recommendations of patients on whom FNAC should be performed for the solid lesions of the thyroid are shown in - Fig. 4. $^{4}$ As the nodule diameter in this case was larger than $20 \mathrm{~mm}$, FNAC was performed as recommended. FNAC is a simple examination that can be performed as an outpatient procedure; however, the frequency of all complications has been reported to be 1.9 to $8.6 \% .^{5}$ Neck swelling is a typical complication, and the causes are rapid diffuse thyroid swelling (dTSaFNA) and hematoma formation because of bleeding. Hematoma is attributed to vascular invasion during puncture; however, the cause of dTSaFNA remains unclear. One theory

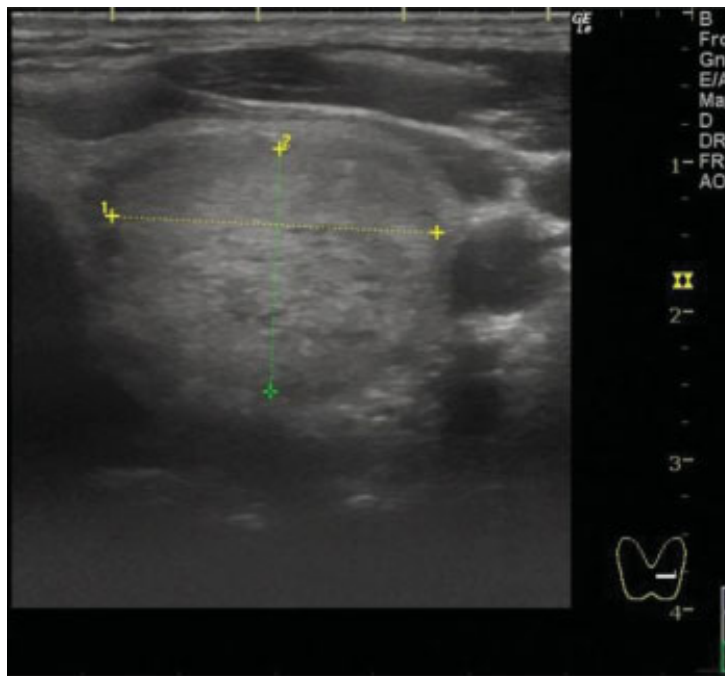

a

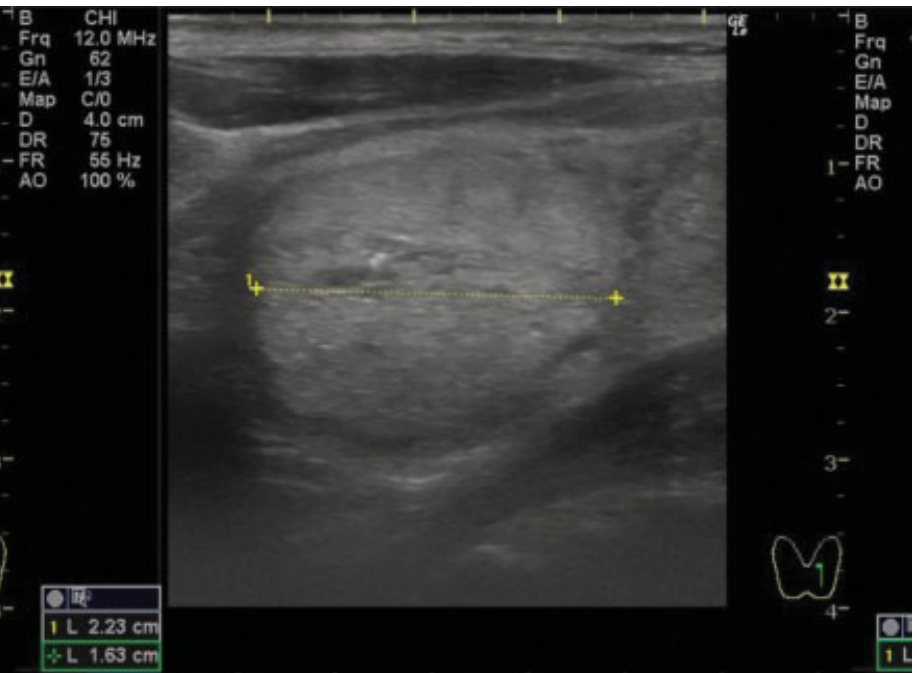

b

Fig. 1 Ultrasonographic findings at the first visit. (a) Horizontal section, (b) sagittal section. A solid mass of $2.23 \times 1.63 \times 2.47 \mathrm{~cm}$. Mosaic-like and microcalcifications were observed inside the mass. 


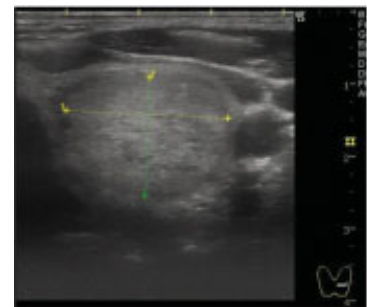

a

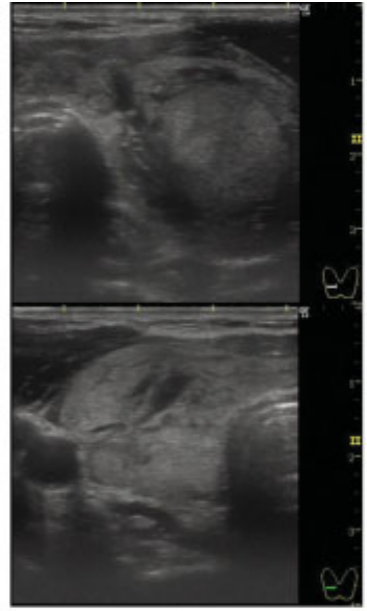

b

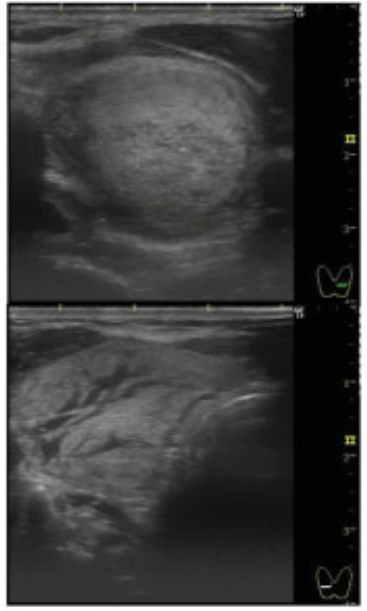

C

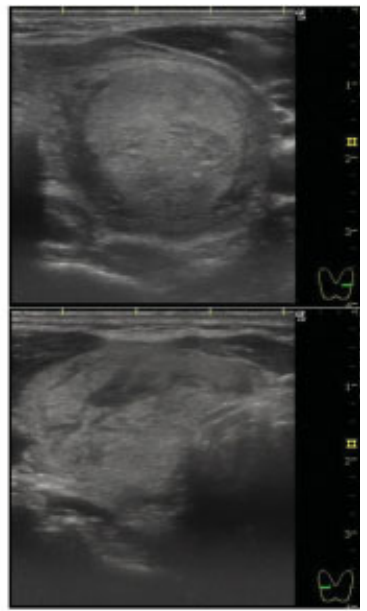

d

Fig. 2 Ultrasonographic findings over time during fine-needle aspiration (FNA) (upper: left lobe; lower: right lobe). (a) Before FNA: a well-defined mass of $22.3 \times 16.3 \times 24.7$ mm was observed. (b) 2 minutes after puncture. Vasodilation around the left lobe mass was prominent. (c) 5 minutes after puncture. Vasodilation of the left lobe became vague and vasodilation of the right lobe became clear. (d) 15 minutes after puncture. Vasodilation of the left lobe became vague.

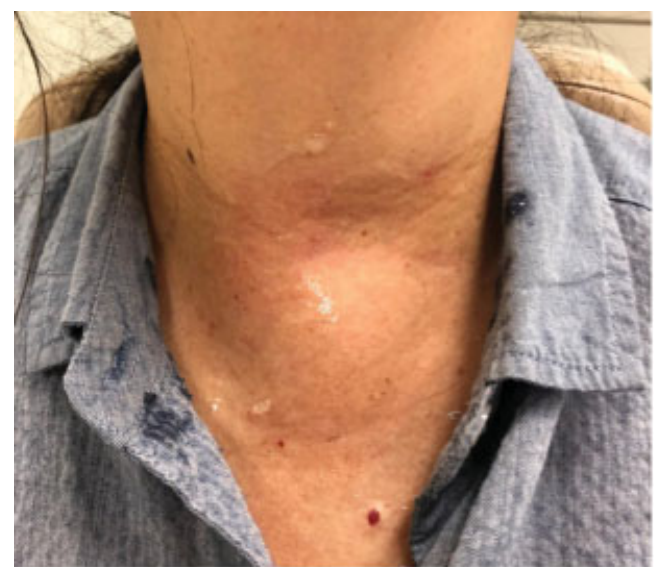

a

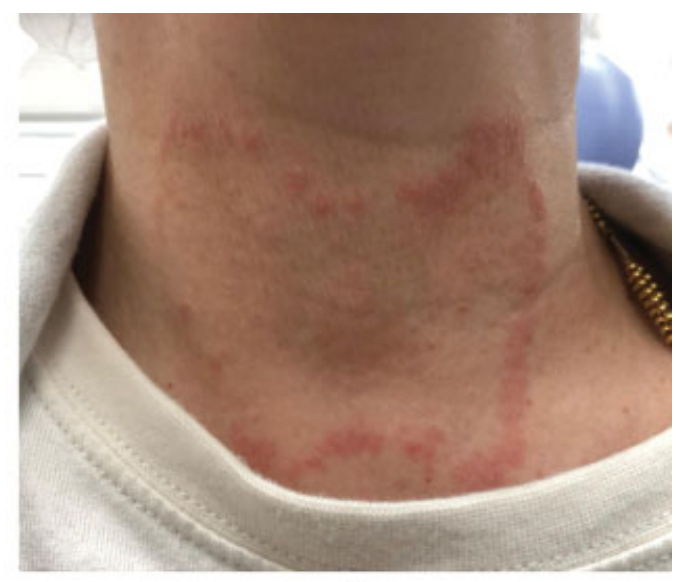

b

Fig. 3 Images of the patient's anterior neck. (a) 15 minutes after the second fine-needle aspiration. The thyroid was diffusely swollen. (b) Five days after the first visit. A wheal matching the marking done with a marker pen appeared.

states that the stimulation during puncture induces substance $\mathrm{P}$ and neurokinin A present at nerve endings in the thyroid, causing the thyroid to swell suddenly and diffusely ${ }^{6}$; however, another theory states that it is caused by the release of calcitonin gene-related peptide, which has a strong vasodilatory effect and is distributed in C cells. ${ }^{2}$ In any case, as neck swelling is caused by vasodilation, many reports have described performing treatment with cooling and steroid administration. Because there are reports of upper airway obstruction in thyroid swelling after puncture because of hematoma, ${ }^{7-10}$ airway assessment over time should be performed even in the case of dTSaFNA. Furthermore, because of these risks, it is considered desirable to obtain written consent before FNA. At our hospital, prior to this case, written consent had not been obtained during FNA; however, since then, examinations have been performed after providing sufficient explanation and obtaining consent. Observation over time using ultrasonography is useful in diagnosing
dTSaFNA. In this case, as shown in - Fig. 1, it could be observed that vasodilation started from around the nodule of the punctured left thyroid lobe and expanded to vasodilation of the contralateral right lobe after some time. At the 15th minute, thyroid swelling continued even though major blood vessels gradually became vague; this could be because dilation occurred in the more peripheral blood vessels. Dilated blood vessels were characterized by poor blood flow; therefore, the combined use of color Doppler imaging may be useful. The time from puncture to swelling was 2 minutes in our case. There have been various reports about swelling occurring from several minutes after, which was a similar result, up to 10 minutes later ${ }^{3}$ or even several hours later. ${ }^{11}$ It is possible that the degree of puncture stimulation, the rate of response to stimulation, or other pathological conditions are not apparent. The most commonly used puncture needles for FNA in Japan are 22 to $23 \mathrm{G}$ needles. In Western countries, however, 25 to $27 \mathrm{G}$ is recommended ${ }^{12}$ to ensure that a sufficient amount of 


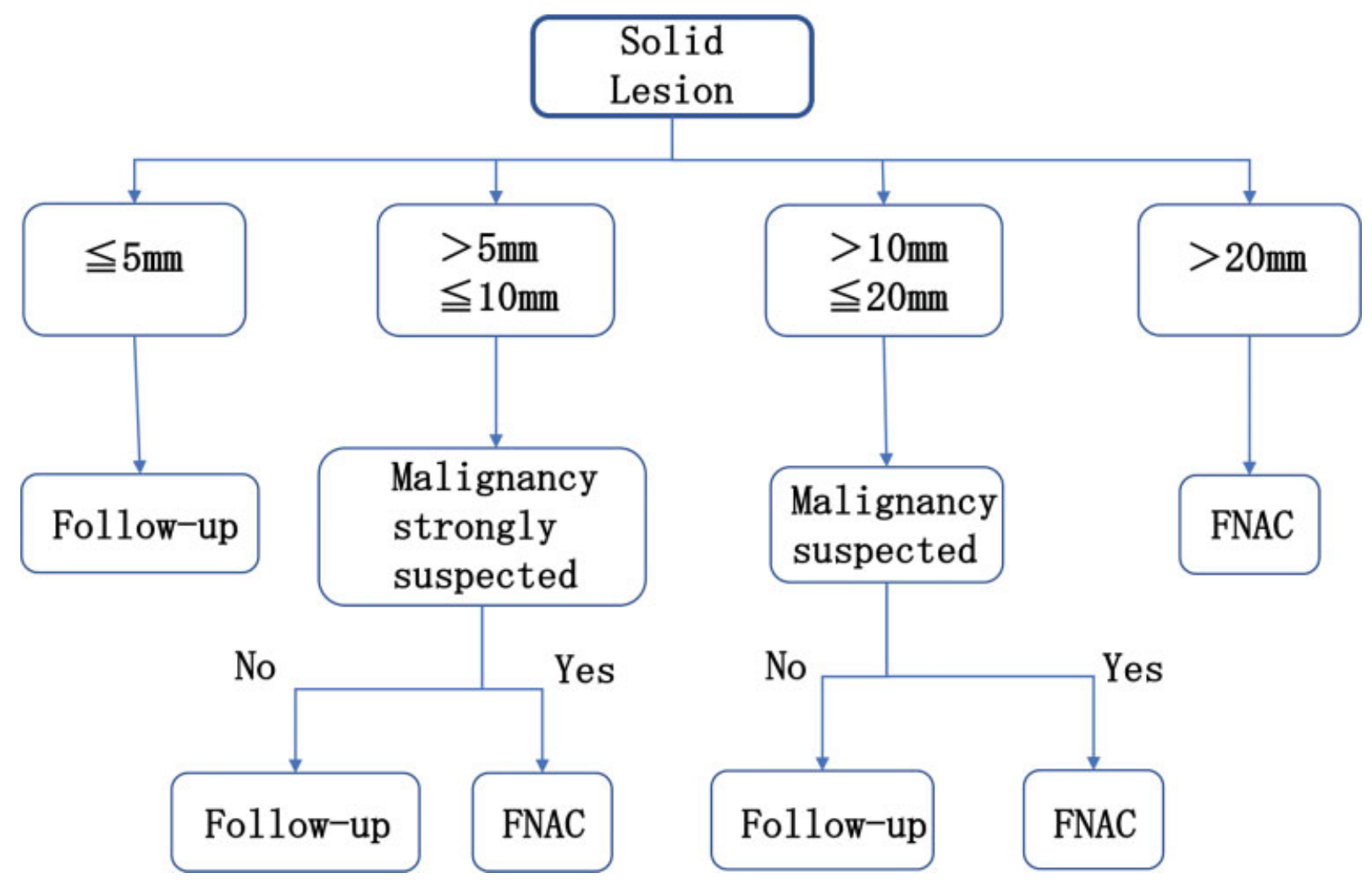

Fig. 4 Ultrasound diagnostic flowchart for solid lesions ${ }^{4}$ (Thyroid Terminology and Diagnostic Criteria Committee. Japan Association of Breast and Thyroid Sonology: Thyroid Ultrasound - A Guidebook for Diagnosis and Management. 3rd revised ed. Tokyo: Nankodo; 2016:50, reproduced with permission from Nankodo). FNAC, fine-needle aspiration cytology.

cells is collected, and pain and bleeding are suppressed. ${ }^{13}$ Furthermore, the sampling rate is better if $24 \mathrm{G}$ is used rather than $20 \mathrm{G}$ and if the needle is rotated rather than not rotated. ${ }^{14}$ FNAC examinations at our hospital are performed by attaching an extension tube to a $22 \mathrm{G}$ needle-having the attendant nurse apply negative pressure with a syringe of $10 \mathrm{~mL}$ after puncture. Despite certain differences depending on the nurse, a negative pressure of approximately 5 to $10 \mathrm{~mL}$ is often applied. A negative pressure of approximately $0.5 \mathrm{~mL}$ is recommended $^{15}$; therefore, the strong aspiration pressure could have caused dTSaFNA. Furthermore, a high diagnostic rate was obtained for thyroid masses even with nonaspiration cytology, which is performed without applying negative pressure ${ }^{16}$; if the stimulus during stimulation causes dTSaFNA, it may be possible to prevent dTSaFNA using a technique that causes less irritation. The patient in this case was originally allergic. A wheal caused by a marker pen (-Fig. 3) was observed, the patient had metal allergies, and the skin at the puncture site had become swollen during injection. The results of a patch test performed at a dermatology clinic at a later date demonstrated strong positivity for Pd and Ir; positivity for Pt, Zn, and $\mathrm{Mn}$; and weak positivity for $\mathrm{Hg}, \mathrm{Cr}$, and $\mathrm{Ni}$. Based on the above, the possibility of an allergic reaction to the puncture needle coated with $\mathrm{Cr}$ and $\mathrm{Ni}$ was initially suspected. However, type I immediate-type allergies because of metal allergies should cause systemic symptoms (such as wheals). Moreover, for type IV allergies represented by allergic contact dermatitis, similar to the wheal caused by a marker pen, reactions do not occur until at least several hours later; therefore, the course does not match this case. Therefore, while we determined that dTSaFNA was highly possible, we cannot deny the possibility that metal allergies might have affected the occurrence of dTSaFNA. Our search of the literature did not reveal any reports of metal allergies causing thyroid swelling after thyroid FNAC. It is anticipated that the causes of dTSaFNA occurrence, including metal allergies, will be elucidated in future.

\section{Conclusions}

We treated a case of dTSaFNA, a rare complication during FNA. Follow-up observations over time using ultrasonography appear to be useful for diagnosis. Note that the abstract of this article was presented at the 81st Annual Meeting of the Society of Practical Otolaryngology (June 27-28, 2019, Nagoya City).

Conflicts of Interest

None declared.

\section{References}

1 Sumiyoshi S, Murakami M, Noguchi H, et al. Hematoma formation and thyroid diffuse swelling after thyroid fine needle aspiration cytology. Endocr J 2011;87:41-43

2 Van den Bruel A, Roelandt P, Drijkoningen M, Hudders JP, Decallonne B, Bouillon R. A thyroid thriller: acute transient and symmetric goiter after fine-needle aspiration of a solitary thyroid nodule. Thyroid 2008;18(01):81-84

3 Ohtsuka Y, Nemoto T, Kunii N, Hanazawa T, Okamoto Y. Three cases of neck swelling after FNAC (fine-needle aspiration cytology). Nippon Jibiinkoka Gakkai Kaiho 2016;119(09):1225-1230 
4 Thyroid Terminology and Diagnostic Criteria Committee. Japan Association of Breast and Thyroid Sonology: Thyroid Ultrasound A Guidebook for Diagnosis and Management. 3rd revised ed. Tokyo: Nankodo; 2016:50

5 Polyzos SA, Anastasilakis AD. Systematic review of cases reporting blood extravasation-related complications after thyroid fine-needle biopsy. J Otolaryngol Head Neck Surg 2010;39(05):532-541

6 Nakagawa Y, Mori K, Hirabayashi S, et al. A case of sudden diffuse thyroid swelling after fine needle aspiration cytology. Endocr J 2007;88:348

7 Tsuzuki N, Kawasaki T, Sahara S, et al. A case of giant goiter with sudden neck swelling after fine needle aspiration cytology, and requiring intubation. Journal of the Japan Broncho-Esophagological Society 2019;70:S79

8 Roh JL. Intrathyroid hemorrhage and acute upper airway obstruction after fine needle aspiration of the thyroid gland. Laryngoscope 2006;116(01):154-156

9 Noordzij JP, Goto MM. Airway compromise caused by hematoma after thyroid fine-needle aspiration. Am J Otolaryngol 2005;26 (06):398-399

10 Park MH, Yoon JH. Anterior neck hematoma causing airway compression following fine needle aspiration cytology of the thyroid nodule: a case report. Acta Cytol 2009;53(01):86-88
11 Chikano S, Kurokawa M, Yamada S, et al. Two cases of sudden diffuse thyroid swelling after fine needle aspiration cytology. Journal of the Japan Otorhinolaryngologists Society 2015; 108:783-790

12 Gharib H, Papini E, Paschke R, et al;AACE/AME/ETA Task Force on Thyroid Nodules. American Association of Clinical Endocrinologists, Associazione Medici Endocrinologi, and European Thyroid Association Medical guidelines for clinical practice for the diagnosis and management of thyroid nodules: executive summary of recommendations. Endocr Pract 2010;16(03):468-475

13 Layfield LJ, Cibas ES, Gharib H, Mandel SJ. Thyroid aspiration cytology: current status. CA Cancer J Clin 2009;59(02):99-110

14 Degirmenci B, Haktanir A, Albayrak R, et al. Sonographically guided fine-needle biopsy of thyroid nodules: the effects of nodule characteristics, sampling technique, and needle size on the adequacy of cytological material. Clin Radiol 2007;62(08): 798-803

15 Yanase Y, Hirokawa M, Maekawa M, et al. Quality control of sample collection and smear method in thyroid fine needle aspiration cytology. J Jpn Soc Clin Cytol 2010;49:431-436

16 Yamada H, Koshikawa T, Suganuma Y, et al. Ultrasound-guided fine needle non-aspiration cytology at thyroid and neck lymph node. J Jpn Soc Clin Cytol 2006;45:84-90 